\title{
Increased reactive oxygen metabolites is associated with cardiovascular risk factors and vascular endothelial damage in middle-aged Japanese subjects
}

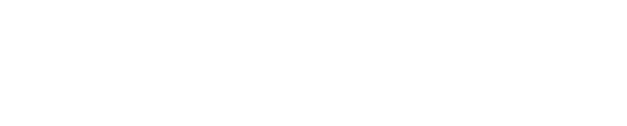

\section{Tomonori Sugiura' \\ Yasuaki Dohi' \\ Hiroyuki Takase ${ }^{2}$ \\ Sumiyo Yamashita' \\ Satoru Tanaka' \\ Genjiro Kimura'}

'Department of Cardio-Renal Medicine and Hypertension, Nagoya City University Graduate School of Medical Sciences, Nagoya, Japan: ${ }^{2}$ Department of Internal Medicine, Enshu Hospital, Hamamatsu, Japan
Correspondence: Tomonori Sugiura Department of Cardio-Renal Medicine and Hypertension, Nagoya City University Graduate School of Medical Sciences, Kawasumi I, Mizuho-cho, Mizuho-ku, Nagoya 467-860I, Japan Tel $+8 \mid 52853822$ I

Fax +8I 528523796

Email tomosugi@med.nagoya-cu.ac.jp
Background: Vascular endothelium, a provider of nitric oxide, is essential for the maintenance of homeostasis in healthy vascular systems. Increased oxidative stress promotes vascular inflammation and is a common pathway involved in endothelial damage. The present study sought to investigate the usefulness of derivative reactive oxygen metabolites (d-ROM) as an oxidative stress marker for detecting endothelial damage in the clinical setting in subjects with early-stage atherosclerosis.

Methods: Study 1 investigated the relationship between serum d-ROM levels and cardiovascular risk factors in apparently healthy middle-aged subjects ( $n=1992,49 \pm 8$ years) who participated in our health checkup program. Study 2 analyzed the association between d-ROM levels and endothelial function assessed by flow-mediated dilation and that between d-ROM levels and highsensitivity $\mathrm{C}$ reactive protein (hs-CRP) levels in middle-aged outpatients with mild-to-moderate cardiovascular risk ( $\mathrm{n}=43,40 \pm 5$ years).

Results: In study 1, the d-ROM level was independently correlated with age, systolic blood pressure, fasting plasma glucose, low-density lipoprotein cholesterol, and brain natriuretic peptide in univariate and multivariate regression analysis. In study 2 , the d-ROM level was correlated positively with the hs-CRP level and inversely with the flow-mediated dilation value. Patients in the highest tertile of d-ROM had significantly lower flow-mediated dilation values compared with patients in the other tertiles. Moreover, after subdivision of patients into four groups according to d-ROM and hs-CRP levels, patients with high levels of both d-ROM and hs-CRP showed significantly reduced flow-mediated dilation as compared with those with low levels of both indices.

Conclusion: The close relationship of d-ROM with cardiovascular risk factors, brain natriuretic peptide, inflammatory markers (hs-CRP), and endothelial function (flow-mediated dilation) suggest that d-ROM is a useful oxidative stress marker for detection of endothelial damage in the clinical setting. Assessment of d-ROM, especially combined with hs-CRP, may be a possible predictor of cardiovascular disease.

Keywords: atherosclerosis, endothelial dysfunction, inflammation, oxidative stress

\section{Introduction}

Cardiovascular disease is a leading cause of mortality and morbidity in most modern societies, with prevention as well as treatment of the condition a focus of recent attention. ${ }^{1}$ Changing lifestyles have highlighted risk factors for cardiovascular disease, including hypertension, dyslipidemia, diabetes mellitus, and smoking. ${ }^{1}$ Because the 
development of cardiovascular disease is largely based on functional and structural changes in systemic vasculature, namely atherosclerosis, early detection of such changes is an important public health issue. ${ }^{1-3}$

Atherosclerosis is a chronic inflammatory disease of the vascular wall initiated by functional alterations in vascular endothelium..$^{2-4}$ Indeed, endothelial dysfunction in resistance vessels, conduit arteries, or coronary arteries independently predicts adverse cardiovascular outcomes, strongly suggesting that endothelial dysfunction is a surrogate marker of cardiovascular events in addition to systemic vascular damage. ${ }^{5-8}$ However, endothelial function cannot be easily assessed in a clinical setting because of technical complexity, and the assessment is limited mainly to research institutes. ${ }^{9-12}$ Therefore, safe, noninvasive, reproducible, and inexpensive modalities are needed to detect and assess endothelial dysfunction, as an initial step in the process of atherosclerosis. ${ }^{13}$

The endothelium produces several bioactive molecules, which could be antiatherogenic or proatherogenic, and homeostasis in a healthy vascular system is maintained by the balance between these factors. ${ }^{3,9}$ Cardiovascular risk factors impair this balance through an increase in oxidative stress, leading to vascular inflammation. ${ }^{9,13}$ Thus, oxidative stress and inflammation are an important part of the endothelial damage process, and laboratory biomarkers of these two states are possible candidates for measuring endothelial function. ${ }^{9,13,14}$ As expected, high-sensitivity $\mathrm{C}$ reactive protein (hs-CRP) is recognized as a reliable predictor of endothelial function and cardiovascular disease. ${ }^{15-17}$ In contrast, markers of oxidative stress, such as malondialdehyde-modified low-density lipoprotein, thiobarbituric acid reactive substances, and 8-isoprostane have not been widely accepted as markers of endothelial function or cardiovascular events. ${ }^{16,18,19}$ Recently, a simple method of detecting hydroperoxide levels by measuring derivatives of reactive oxygen metabolites (d-ROM) was developed. ${ }^{20-22}$ Thus, the present study was designed to test our fundamental hypothesis that d-ROM is a useful marker of oxidative stress for evaluating cardiovascular risk burden and for detecting endothelial damage in the clinical setting.

\section{Methods}

The present study comprised two parallel substudies, ie, one evaluating the relationship between d-ROM and cardiovascular risk factors in the general population (study 1) and the other investigating the impact of markers of oxidative stress on endothelial function in subjects with mild-to-moderate cardiovascular risk factors (study 2).

\section{Study I}

Middle-aged subjects (aged 20-60 years) who underwent health checkups at Enshu Hospital from September 2008 to August 2009 were enrolled. In addition to our standard health checkup program (an interview regarding health status, routine physical examination, chest x-ray, electrocardiography, brain natriuretic factor measurement, and laboratory assessment of cardiovascular risk factors), serum levels of d-ROM were measured. Subjects with obvious organ damage, a complication of malignant neoplasm or active inflammatory disease, and/or a history of cardiovascular events were excluded. Current smokers were also excluded, because serum reactive oxygen metabolite levels are influenced by smoking. ${ }^{23}$ Blood pressure was measured using a standard mercury sphygmomanometer with the subject in the sitting position. The relationship between serum reactive oxygen metabolites and cardiovascular risk factors was investigated. The study protocol was approved by the ethics committee of Enshu Hospital, and written informed consent was obtained from all subjects before the study commenced.

\section{Study 2}

Middle-aged never-treated outpatients who were newly diagnosed with hypertension or dyslipidemia at Nagoya City University Hospital from September 2008 to August 2009 , with one or more additional risk factors, including hypertension, dyslipidemia, and smoking, namely mild-to-moderate cardiovascular risk according to the guidelines of the Japan Atherosclerosis Society and Japanese Society of Hypertension, were enrolled in this second study. ${ }^{24,25}$ Patients with severe cardiovascular risk, obvious organ damage, diabetes mellitus, a history of cardiovascular events, a complication of malignant neoplasm or active inflammatory disease, and/or taking any medications influencing vascular integrity were excluded. The study examination was performed before the start of medication and patients who smoked were instructed to stop smoking 24 hours before the examination. Endothelial function was assessed by investigating endothelium-dependent flowmediated vasodilation in a fasting condition. ${ }^{12,13}$ In addition to the laboratory measurements including hs-CRP and d-ROM, circulating endothelial progenitor cells, which are considered to mobilize into the peripheral circulation from the bone marrow and play a pivotal role in repairing endothelial damage, were evaluated by measuring CD34-positive progenitor cells. ${ }^{26-28}$ Blood pressure was measured in the nondominant arm using a validated oscillometric technique (HEM-7070; Omron Corporation, Kyoto, Japan) in a seated position. 
Relationships between serum reactive oxygen metabolites and flow-mediated dilation values, hs-CRP, and the number of circulating endothelial progenitor cells were investigated. The study protocol was approved by the ethics committee of Nagoya City University Graduate School of Medical Sciences, and written informed consent was obtained from each subject prior to starting the study.

\section{Biochemical analysis}

Serum total cholesterol, low-density lipoprotein cholesterol, high-density lipoprotein cholesterol, and triglyceride levels were determined by standard laboratory assays. Brain natriuretic peptide was measured by radioimmunoassay (Shionoria BNP kit, Shionogi, Osaka, Japan). Hs-CRP was determined by latex-enhanced immunonephelometry on a BN II analyzer (Dade Behring, Marburg, Germany).

The d-ROM level was measured as previous reported. ${ }^{20-22}$ Briefly, serum sample and acidic buffered solution (R2 kit reagent, $\mathrm{pH} 4.8$ ) were mixed in a cuvette, and a chromogenic substrate (R1 kit reagent) was then added to the cuvette. After mixing well, the cuvette was immediately incubated in the thermostatic block of the analyzer for five minutes, and then absorbance at $505 \mathrm{~nm}$ was recorded. Measurements are expressed as arbitrary units (Carr). This method spectrophotometrically detects the oxidization of N,N-diethyl-paraphenylenediamine by radicals converted from hydroperoxides. The intra-assay and interassay coefficients of variation for d-ROM were $3.2 \%$ and $3.6 \%$, respectively, and the analytical range was 50-500 Carr. The normal reference level indicated by the manufacturer is 250-300 Carr.

Circulating CD34-positive mononuclear cells, one form of circulating endothelial progenitor cells, were defined as $\mathrm{CD} 34^{+}$progenitor cells and quantified by flow cytometry as described previously. ${ }^{26}$ In detail, $4 \mathrm{~mL}$ of peripheral blood was drawn and white blood cells were stained with peridinin chlorophyll-a protein-conjugated anti-CD45 monoclonal antibody (Becton Dickinson, Franklin Lakes, NJ) and fluorescein isothiocyanate-conjugated anti-CD34 monoclonal antibody (IOTest; Beckman Coulter, Tokyo, Japan). Samples were subjected to two-dimensional side scatter-fluorescence dot plot analysis (FACScan). After appropriate gating with low cytoplasmic granularity and low expression of CD45, the number of $\mathrm{CD}^{+} 4^{+}$progenitor cells was quantified and expressed as number of cells per $10^{6}$ total events.

\section{Determination of endothelial function}

The flow-mediated dilation technique is a noninvasive method for assessment of endothelial function. ${ }^{24}$
Flow-mediated dilation was measured using high resolution ultrasound apparatus with a $7.5 \mathrm{mHz}$ linear array transducer (ProSound SSD-6500SV; Aloka Co Ltd, Tokyo, Japan) equipped with an ECG-gated computer system having automatic edge detection software (e-Tracking system, Aloka Co Ltd, Tokyo, Japan) according to the guidelines of the International Brachial Artery Reactivity Task Force. ${ }^{12,13}$ Endothelial function was measured in the supine position with the brachial artery diameter of the nondominant arm at $5 \mathrm{~cm}$ above the elbow in a dark, quiet, and air-conditioned room $\left(22^{\circ} \mathrm{C}-25^{\circ} \mathrm{C}\right)$. The longitudinal image of the brachial artery was continuously recorded throughout the measurements. The e-Tracking system enables continuous and stable image acquisition. Baseline data was recorded for one minute after 15 minutes of supine rest and a sphygmomanometric cuff placed around the forearm distal to the cubital fossa was then inflated to up to either $200 \mathrm{mmHg}$ or $50 \mathrm{mmHg}$ above each subject's systolic blood pressure to occlude arterial inflow. After a period of five minutes, subsequent cuff deflation causes dilation of brachial artery by an increase in shear stress. The diameter of the brachial artery was continuously recorded for five minutes, and flow-mediated dilation was automatically calculated as percent change in peak vessel diameter from the baseline. With 15 minutes of rest after acquisition of endotheliumdependent vasodilation, this parameter was subsequently quantified. After acquisition of baseline data for one minute, $0.15 \mathrm{mg}$ of glyceryl trinitrate (Nitropen ${ }^{\mathrm{TM}}$; Nihonkayaku, Tokyo, Japan) was administered sublingually and the diameter of the brachial artery was continuously recorded for five minutes and analyzed by a similar method. The intraobserver coefficient of variation for the baseline diameter was $2.5 \%$. All subjects were examined by a single cardiologist. Both flow-mediated dilation and glyceryl trinitrate-induced endothelium-independent dilation were expressed as the percent change from baseline.

\section{Statistical analysis}

Data were analyzed using StatView 5.0 (SAS Institute, Cary, NC) and expressed as means \pm standard deviation. Urinary albumin was expressed as urinary albumin to creatinine ratio (mg/g creatinine). Because urinary albumin to creatinine ratio, brain natriuretic peptide, and hs-CRP did not show a normal distribution, the value was analyzed after log-transformation. The dichotomous variables (gender and smoking status) were assigned values of 0 (female and nonsmoking) and 1 (male and smoking). Univariate and multivariate linear regression analyses associated with d-ROM level or flow-mediated 
dilation values were performed. Comparisons among multiple subgroups were performed using one-way analysis of variance followed by Bonferroni's post hoc test. Statistical significance was set at $P<0.05$.

\section{Results}

\section{Study I}

Table 1 details the characteristics of subjects in study 1 . The mean age was 49 years and the mean blood pressure was 122/75 mmHg. There were 226 (11\%), 756 (38\%), and $86(4 \%)$ subjects with hypertension, dyslipidemia, and diabetes mellitus, respectively, indicating that the study subjects represented a general population. The d-ROM value in subjects without cardiovascular risk was $298 \pm 50$ Carr. In addition to known cardiovascular risk parameters (age, gender, systolic blood pressure, hemoglobin, creatinine, fasting plasma glucose, uric acid, and lipid parameters [total cholesterol, low-density lipoprotein cholesterol, and high-density lipoprotein cholesterol]), brain natriuretic peptide showed significant correlations with d-ROM by univariate regression analysis $(\mathrm{r}=0.059$, $P<0.05$, Table 1). Multivariate regression analysis showed that d-ROM was independently associated with age $(P<0.001)$, gender $(P<0.0001)$, systolic blood pressure $(P<0.05)$, fasting plasma glucose $(P<0.05)$, low-density lipoprotein cholesterol ( $P<0.05$ ), and brain natriuretic peptide $(P<0.05$, Table 2$)$.

\section{Study 2}

To evaluate a possible association of d-ROM with endothelial function, flow-mediated dilation values, $\mathrm{CD} 34^{+}$progenitor cell number, and hs-CRP levels were assessed in subjects with mild-to-moderate risk. Characteristics of 43 patients involved in this series of the study are shown in Table 3. The mean age was 40 years and the mean d-ROM level was 314 Carr, which was younger and lower, respectively, than subjects in Study 1. In subjects with mild-to-moderate risk, the d-ROM level correlated significantly with hs-CRP levels $(\mathrm{r}=0.37, P<0.05)$ and flow-mediated dilation values ( $\mathrm{r}=-0.40, P<0.01$ ), but not with the $\mathrm{CD} 34^{+}$progenitor cell number or glyceryl trinitrate-induced endotheliumindependent dilation values (Figure 1). To confirm the impact of d-ROM on hs-CRP and flow-mediated dilation, patients were divided into tertiles according to d-ROM levels (cut off levels 295 and 335 Carr). Patients in the highest tertile (>335 Carr) had a significantly increased hs-CRP level and a reduced flow-mediated dilation value compared with those in the lowest tertile $(P<0.05$, Figure 2$)$.

In a separate analysis, patients were divided into four subgroups according to d-ROM and hs-CRP levels to compare the impact of oxidative stress markers and inflammation status on flow-mediated dilation. The mean value of d-ROM (316 Carr) and the hs-CRP value $(0.50 \mathrm{mg} / \mathrm{L})$ corresponding to the mean $\log$ (hs-CRP) value were used as cutoff values. This analysis revealed that the flow-mediated

Table I Characteristics of subjects and results of univariate regression analysis demonstrating the relationship of variables with d-ROM in study I

\begin{tabular}{|c|c|c|c|}
\hline Variable & Total subjects $(n=1992)$ & Coefficient ( $r$ ) & $P$ value \\
\hline Age (years) & $49 \pm 8$ & 0.17 & $<0.0001$ \\
\hline Gender, male (\%) & $800(40)$ & -0.32 & $<0.0001$ \\
\hline BMI $\left(\mathrm{kg} / \mathrm{m}^{2}\right)$ & $22.4 \pm 3.2$ & 0.038 & 0.088 \\
\hline Systolic BP (mmHg) & $122 \pm 15$ & 0.079 & $<0.001$ \\
\hline Diastolic BP (mmHg) & $75 \pm 10$ & 0.029 & 0.19 \\
\hline Hemoglobin (g/dL) & $13.8 \pm 1.2$ & -0.22 & $<0.0001$ \\
\hline Creatinine $(\mathrm{mg} / \mathrm{dL})$ & $0.72 \pm 0.15$ & -0.25 & $<0.0001$ \\
\hline UACR (mg/g creatinine) & $6.5 \pm 22.5$ & 0.098 & $<0.0001$ \\
\hline Uric acid (mg/dL) & $5.0 \pm 1.3$ & -0.15 & $<0.0001$ \\
\hline FPG (mg/dL) & $95 \pm 15$ & 0.055 & $<0.05$ \\
\hline Total C (mg/dL) & $211 \pm 33$ & 0.112 & $<0.0001$ \\
\hline LDL-C (mg/dL) & $122 \pm 29$ & 0.096 & $<0.0001$ \\
\hline HDL-C (mg/dL) & $63 \pm 14$ & 0.087 & $<0.000$ I \\
\hline Triglycerides (mg/dL) & $98 \pm 60$ & -0.029 & 0.20 \\
\hline BNP (pg/mL) & $13.8 \pm 14.3$ & 0.059 & $<0.05$ \\
\hline d-ROM (Carr) & $320 \pm 61$ & - & - \\
\hline
\end{tabular}

Note: Data are expressed as means \pm standard deviation.

Abbreviations: d-ROM, derivative reactive oxygen metabolites; BMI, body mass index, BP; blood pressure; UACR, urinary albumin to creatinine ratio; FPG, fasting plasma glucose; total C, total cholesterol; LDL-C, low-density lipoprotein cholesterol; HDL-C, high-density lipoprotein cholesterol; BNP, brain natriuretic peptide. 
Table 2 Multivariate regression analysis demonstrating factors associated with d-ROM in study I

\begin{tabular}{|c|c|c|c|c|}
\hline Variables & $\beta$ & SE & Standardized coefficient & $P$ \\
\hline Age (years) & 0.70 & 0.18 & 0.096 & $<0.001$ \\
\hline BMI $\left(\mathrm{kg} / \mathrm{m}^{2}\right)$ & 0.80 & 0.51 & 0.044 & 0.099 \\
\hline Gender male & -39 & 5.3 & -0.31 & $<0.000$ I \\
\hline Systolic BP (mmHg) & 0.24 & 0.10 & 0.060 & $<0.05$ \\
\hline Hemoglobin (g/dL) & -0.95 & 1.8 & -0.018 & 0.60 \\
\hline Creatinine $(\mathrm{mg} / \mathrm{dL})$ & -12 & 13 & -0.031 & 0.35 \\
\hline UACR (mg/g creatinine) & 2.8 & 3.6 & 0.017 & 0.45 \\
\hline Uric acid (mg/dL) & 1.8 & 1.4 & 0.038 & 0.20 \\
\hline FPG (mg/dL) & 0.20 & 0.096 & 0.050 & $<0.05$ \\
\hline LDL-C (mg/dL) & 0.11 & 0.052 & 0.050 & $<0.05$ \\
\hline HDL-C (mg/dL) & 0.17 & 0.11 & 0.039 & 0.13 \\
\hline Triglycerides (mg/dL) & 0.016 & 0.028 & 0.015 & 0.55 \\
\hline $\mathrm{BNP}(\mathrm{pg} / \mathrm{mL})$ & -11 & 5.7 & -0.048 & $<0.05$ \\
\hline
\end{tabular}

Abbreviations: SE, standard error; d-ROM, derivative reactive oxygen metabolites; BMI, body mass index; BP, blood pressure; UACR, urinary albumin to creatinine ratio; FPG, fasting plasma glucose; LDL-C, low-density lipoprotein cholesterol; HDL-C, high-density lipoprotein cholesterol; BNP, brain natriuretic peptide.

dilation value was significantly reduced in the subgroup with higher levels of both d-ROM and hs-CRP than in the subgroup with lower levels of both d-ROM and hs-CRP $(P<0.05$, Figure 3$)$.

Table 3 indicates the relationships between the flowmediated dilation value and other parameters. The flowmediated dilation value was correlated inversely with age ( $\mathrm{r}=-0.31, P<0.01)$, smoking status $(\mathrm{r}=-0.36, P<0.05)$, triglycerides $(\mathrm{r}=-0.40, P<0.01)$, hs-CRP $(\mathrm{r}=-0.34$, $P<0.05)$, and d-ROM (r $=-0.42, P<0.01)$, and positively with high-density lipoprotein cholesterol $(\mathrm{r}=0.37, P<0.05)$ and $\mathrm{CD}_{34}{ }^{+}$progenitor cells $(\mathrm{r}=0.32, P<0.05)$.

\section{Discussion}

The present study revealed that classical cardiovascular risk parameters (age, systolic blood pressure, fasting plasma glucose, and low-density lipoprotein cholesterol) independently predicted the d-ROM value, that d-ROM levels were positively correlated with hs-CRP levels and inversely correlated with the flow-mediated dilation value,

Table 3 Characteristics of patients enrolled in study 2 and correlation of factors associated with FMD

\begin{tabular}{|c|c|c|c|}
\hline Variable & Total patients $(n=43)$ & Coefficient ( $r$ ) & $P$ value \\
\hline Age (years) & $40 \pm 5$ & -0.31 & $<0.05$ \\
\hline BMI $\left(\mathrm{kg} / \mathrm{m}^{2}\right)$ & $24.5 \pm 3.4$ & -0.21 & 0.19 \\
\hline Gender male (\%) & 39 (91\%) & 0.28 & 0.07 \\
\hline Smoking status (\%) & II (26\%) & -0.36 & $<0.05$ \\
\hline Systolic BP (mmHg) & $134 \pm 12$ & -0.22 & 0.17 \\
\hline Diastolic BP (mmHg) & $86 \pm 9$ & -0.10 & 0.55 \\
\hline Hemoglobin (g/dL) & $15.3 \pm 1.1$ & -0.094 & 0.56 \\
\hline Creatinine (mg/dL) & $0.77 \pm 0.13$ & -0.010 & 0.95 \\
\hline FPG (mg/dL) & $96 \pm 7$ & -0.27 & 0.25 \\
\hline $\mathrm{HbA}_{\mathrm{Ic}}(\%)$ & $5.0 \pm 0.5$ & -0.19 & 0.45 \\
\hline Total C (mg/dL) & $205 \pm 33$ & -0.091 & 0.57 \\
\hline LDL-C (mg/dL) & $122 \pm 29$ & -0.11 & 0.48 \\
\hline HDL-C (mg/dL) & $55 \pm 12$ & 0.37 & $<0.05$ \\
\hline Triglycerides (mg/dL) & $153 \pm 93$ & -0.40 & $<0.01$ \\
\hline hs-CRP (mg/L) & $0.87 \pm 1.1$ & -0.34 & $<0.05$ \\
\hline d-ROM (Carr) & $316 \pm 45$ & -0.42 & $<0.01$ \\
\hline CD34+ PC (106/cells) & $326 \pm 172$ & 0.32 & $<0.05$ \\
\hline FMD (\%) & $4.0 \pm 1.7$ & - & - \\
\hline GTD (\%) & $15.6 \pm 5.3$ & - & - \\
\hline
\end{tabular}

Note: Data are expressed as means \pm standard deviation.

Abbreviations: FMD, flow-mediated dilation; BMI, body mass index; BP, blood pressure; FPG, fasting plasma glucose; total C, total cholesterol; LDL-C, low-density lipoprotein cholesterol; HDL-C, high-density lipoprotein cholesterol; hs-CRP, high-sensitivity C reactive protein; d-ROM, derivative reactive oxygen metabolites; CD34+ PC, CD34-positive progenitor cells; GTD, glyceryl trinitrate-induced dilation. 


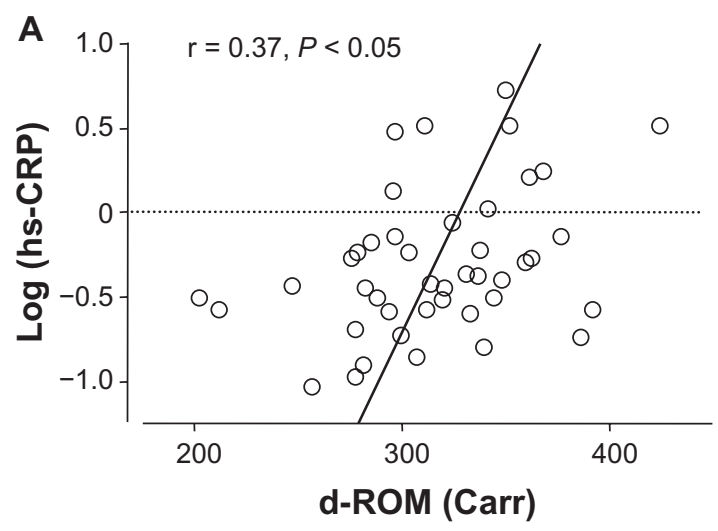

B

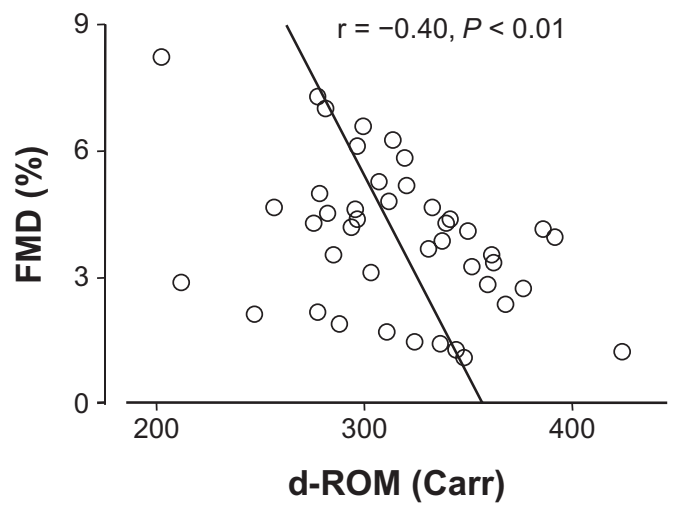

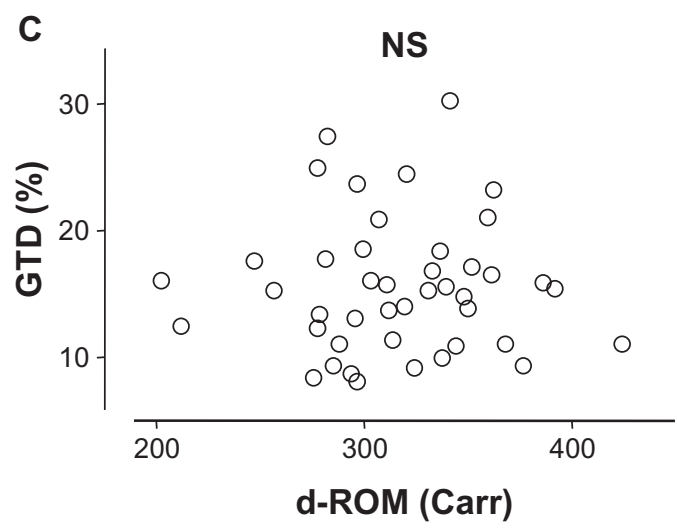

D

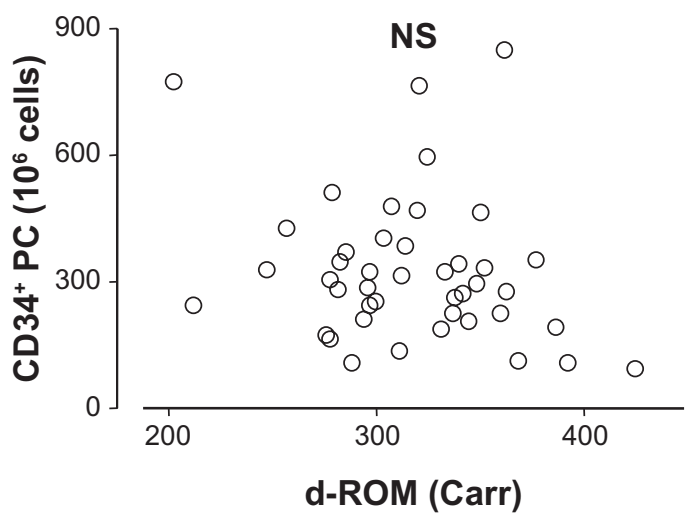

Figure I Relationship of derivative reactive oxygen metabolites (d-ROM) level with (A) high-sensitivity C reactive protein (hs-CRP) levels, (B) flow-mediated dilation (FMD) values, (C) glyceryl trinitrate-induced dilation (GTD), and (D) CD34-positive progenitor cell (CD34+ $P C)$ number in study 2 ( $n=43$ ).

and that subjects with high levels of both d-ROM and hs-CRP had significantly reduced flow-mediated dilation values compared with subjects with low levels of both markers of oxidative stress and inflammation. These findings show that d-ROM is a useful biomarker for detecting endothelial dysfunction, and is thus a surrogate marker of vascular failure and cardiovascular events. To the best of our knowledge, this is the first study that demonstrates a significant and close relationship between d-ROM and vascular function.
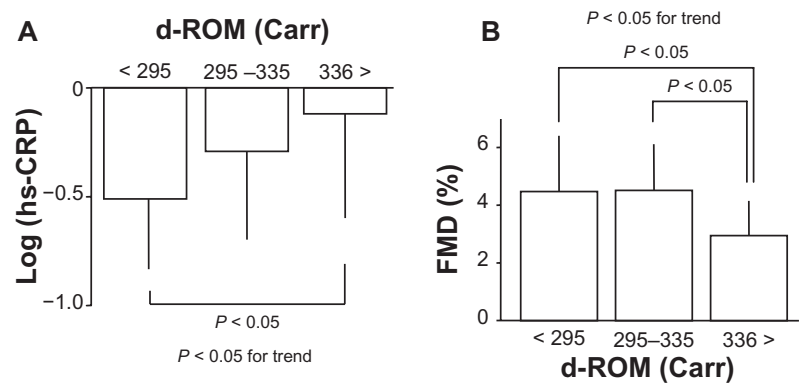

Figure 2 Impact of derivative reactive oxygen metabolites (d-ROM) on highsensitivity $C$ reactive protein (hs-CRP; panel $(\mathbf{A})$ ) levels and flow-mediated dilation (FMD; panel (B)) in study $2(n=43)$. Patients were divided into tertiles according to d-ROM levels. Data are expressed as means \pm standard deviation.
High levels of d-ROM, especially combined with high hs-CRP, seem to indicate a significant deterioration of endothelial function.

Oxidative stress associated with and/or derived from cardiovascular risk factors, including dyslipidemia, hypertension, and smoking, is implicated in the pathogenesis of vascular damage and contributes to the development of atherosclerosis. ${ }^{6,716}$ However, a marker of oxidative stress suitable for assessment of early-stage atherosclerosis has not been established. The present study demonstrated a significant association between d-ROM and several conventional cardiovascular risk parameters in the general population, suggesting that the d-ROM level reflects total cardiovascular risk in individuals. Furthermore, endothelial function progressively deteriorated with increasing d-ROM levels in subjects with mild-to-moderate cardiovascular risk. Similar flow-mediated dilation values in the first and second tertiles of d-ROM in study 2 suggest that there is a threshold at which oxidative stress causes detectable endothelial dysfunction. Alternatively, the results may reflect the concept that the endothelial function is determined not only by offending endothelial factors, such as oxidative stress, but also by the 


\section{$P<0.05$ for trend}

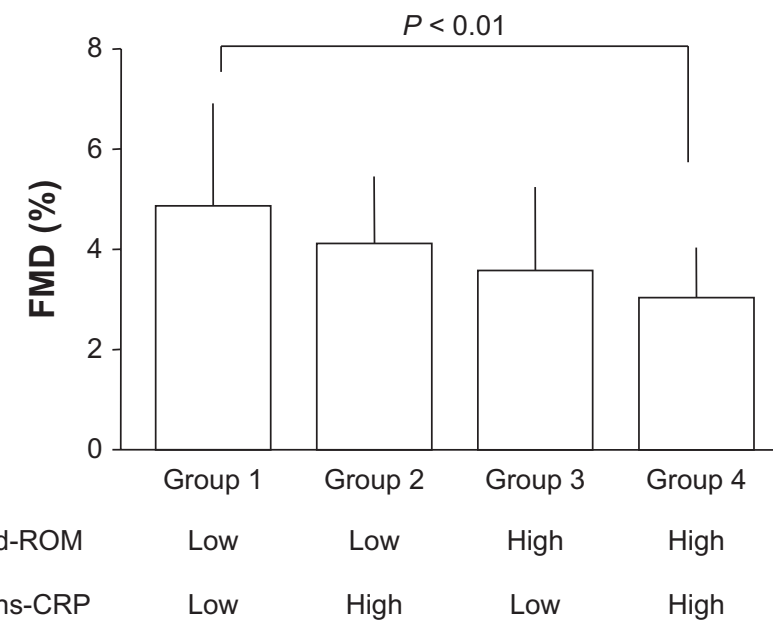

Figure 3 Impact of a combination of derivative reactive oxygen metabolites (d-ROM) and high-sensitivity $\mathrm{C}$ reactive protein (hs-CRP) on flow-mediated dilation (FMD) in study $2(n=43)$. Patients were subdivided into four groups according to the d-ROM level (cutoff level is 316 Carr, mean value) and the hs-CRP level [cutoff level is $0.50 \mathrm{mg} / \mathrm{L}$, corresponding to the mean value of log (hs-CRP)]. Data are expressed as means \pm standard deviation.

balance between offending and defending factors. Because subjects in study 2 were assumed to have subclinical atherosclerosis, d-ROM may be a sensitive marker of endothelial damage induced by oxidative stress in the early stages of atherosclerosis. On the other hand, markers of oxidized modification of lipid components are useful, particularly for the assessment of advanced atherosclerosis. ${ }^{29}$ Indeed, the malondialdehyde-modified low-density lipoprotein level has been shown to be increased independently of low-density lipoprotein cholesterol levels in patients with coronary artery disease, especially in patients with unstable plaques. ${ }^{30-32}$

Although we did not compare the clinical value of d-ROM with other oxidative stress markers, the demonstrated close correlation of d-ROM and hs-CRP, an established predictor of cardiovascular events, ${ }^{17,21,22}$ suggested it to be a reliable marker for cardiovascular risk assessment. Because d-ROM and hs-CRP are fundamentally different in nature, the measurements of these two markers may be complementary. Indeed, measuring a combination of d-ROM and hs-CRP would provide powerful and useful information in subjects with concealed high risk, because an increase in both hsCRP and d-ROM levels, but not in either of the markers, predicts definite endothelial dysfunction in subjects with mild-to-moderate cardiovascular risk. In this context, both d-ROM and hs-CRP are suitable for screening of individual cardiovascular risk in early-stage atherosclerosis.

The present results demonstrated that d-ROM was independently correlated with brain natriuretic peptide as well as classical cardiovascular risk factors. Because brain natriuretic peptide is broadly accepted as a predictor of cardiac function, cardiac load, and cardiac events, ${ }^{33,34}$ our findings indicate that oxidative stress is closely associated with functional and structural alteration of the heart. Accumulation of cardiovascular risk may affect the circulatory system including the vasculature and heart through an increase in oxidative stress, and d-ROM may reflect these changes.

Recently, several studies have confirmed a significant correlation between the number and activity of circulating endothelial progenitor cells and endothelial function. ${ }^{26-28}$ In one study, a reduction in circulating endothelial progenitor cell number was associated with an increased risk of cardiovascular disease. ${ }^{27}$ In the present study, the number of CD $34^{+}$ progenitor cells was correlated with the flow-mediated dilation value, but not with d-ROM. Assessment of other cell fractions or function, such as migration, proliferation, and antioxidant properties, may be essential for evaluating circulating endothelial progenitor cells, although the complicated and specialized investigations needed might not be amenable to use in the clinical setting. ${ }^{26,28}$

The present study had several limitations. First, sample size for the evaluation of endothelial function was small, and heterogeneity in background of the subjects may have affected the results. Further study with increased subject numbers is therefore needed to provide a definitive conclusion. Second, the impact of smoking, one of the most important risk factors, on d-ROM was not assessed in the general population (study 1). Third, although patients with the smoking habit in study 2 were instructed to stop smoking 24 hours before the examination, it has not been established that the acute effects of smoking disappeared within 24 hours. Fourth, observed correlations between d-ROM and endothelial function or other variables do not necessary mean causal relationships, and the predictive value of the index of oxidative stress could not be evaluated in the present cross-sectional study. Fifth, we could not identify the source of oxidative stress measured by d-ROM. Further biological assessments to elucidate the underlying mechanisms and interventional studies are necessary to confirm the clinical significance of the index.

In conclusion, increased oxidative stress evaluated by d-ROM was significantly associated with cardiovascular risk factors, systemic inflammation, and endothelial dysfunction. Assessment of d-ROM, which is easy to measure without any technical complexity, is a useful and reliable way to detect concealed endothelial damage in the clinical setting and may be a powerful predictor of cardiovascular disease. 


\section{Disclosure}

The authors report no conflicts of interest in this work.

\section{References}

1. Mozaffarian D, Wilson PW, Kannel WB. Beyond established and novel risk factors: lifestyle risk factors for cardiovascular disease. Circulation. 2008;117(23):3031-3038.

2. Ross R. Atherosclerosis: an inflammatory disease. N Engl J Med. 1999; 340(2):115-126.

3. Vanhoutte PM, Shimokawa H, Tang EH, Feletou M. Endothelial dysfunction and vascular disease. Acta Physiol. 2009;196(2):193-222.

4. Libby P. Inflammation in atherosclerosis. Nature. 2002;420(6917): 868-874.

5. Perticone F, Ceravolo R, Pujia A, et al. Prognostic significance of endothelial dysfunction in hypertensive patients. Circulation. 2001;104(2):191-196.

6. Halcox JP, Schenke WH, Zalos G, et al. Prognostic value of coronary vascular endothelial dysfunction. Circulation. 2002;106(6):653-658.

7. Davignon J, Ganz P. Role of endothelial dysfunction in atherosclerosis. Circulation. 2004;109(23 Suppl 1):III27-III32.

8. Katz SD, Hryniewicz K, Hriljac I, et al. Vascular endothelial dysfunction and mortality risk in patients with chronic heart failure. Circulation. 2005;111(3):310-314.

9. Deanfield JE, Halcox JP, Rabelink TJ. Endothelial function and dysfunction: testing and clinical relevance. Circulation. 2007;115(10): $1285-1295$.

10. Corretti MC, Anderson TJ, Benjamin EJ, et al. International brachial artery reactivity task force. Guidelines for the ultrasound assessment of endothelial-dependent flow-mediated vasodilation of the brachial artery: a report of the international brachial artery reactivity task force. J Am Coll Cardiol. 2002;39(2):257-265.

11. Bots ML, Westerink J, Rabelink TJ, de Koning EJ. Assessment of flow-mediated vasodilatation (FMD) of the brachial artery: effects of technical aspects of the FMD measurement on the FMD response. Eur Heart J. 2005;26(4):363-368.

12. Widlansky ME, Gokce N, Keaney JF Jr, Vita JA. The clinical implications of endothelial dysfunction. $J$ Am Coll Cardiol. 2003;42(7): $1149-1160$

13. Cai H, Harrison DG. Endothelial dysfunction in cardiovascular diseases: the role of oxidant stress. Circ Res. 2000;87(10):840-844.

14. Higashi Y, Noma K, Yoshizumi M, Kihara Y. Endothelial function and oxidative stress in cardiovascular diseases. Circ J. 2009;73(3): 411-418.

15. Ridker PM, Brown NJ, Vaughan DE, Harrison DG, Mehta JL. Established and emerging plasma biomarkers in the prediction of first atherothrombotic events. Circulation. 2004;109(25 Suppl 1):IV6-IV19.

16. Dohi Y, Takase H, Sato K, Ueda R. Association among C-reactive protein, oxidative stress, and traditional risk factors in healthy Japanese subjects. Int J Cardiol. 2007;115(1):63-66.

17. Blake GJ, Ridker PM. C-reactive protein and other inflammatory risk markers in acute coronary syndromes. $J$ Am Coll Cardiol. 2003;41 (4 Suppl S):37S-42S.

18. Walter MF, Jacob RF, Jeffers B, et al; PREVENT study. Serum levels of thiobarbituric acid reactive substances predict cardiovascular events in patients with stable coronary artery disease: a longitudinal analysis of the PREVENT study. J Am Coll Cardiol. 2004;44(10):1996-2002.

Vascular Health and Risk Management

\section{Publish your work in this journal}

Vascular Health and Risk Management is an international, peerreviewed journal of therapeutics and risk management, focusing on concise rapid reporting of clinical studies on the processes involved in the maintenance of vascular health; the monitoring, prevention and treatment of vascular disease and its sequelae; and the involvement of
19. Kiechl S, Willeit J, Mayr M, et al. Oxidized phospholipids, lipoprotein(a), lipoprotein-associated phospholipase A2 activity, and 10-year cardiovascular outcomes: prospective results from the Bruneck study. Arterioscler Thromb Vasc Biol. 2007;27(8):1788-1795.

20. Cesarone MR, Belcaro G, Carratelli M, et al. A simple test to monitor oxidative stress. Int Angiol. 1999;18(2):127-130.

21. Kamezaki F, Yamashita K, Kubara T, et al. Derivatives of reactive oxygen metabolites correlates with high-sensitivity C-reactive protein. J Atheroscler Thromb. 2008;15(4):206-212.

22. Hirose H, Kawabe H, Komiya N, Saito I. Relations between serum reactive oxygen metabolites (ROMs) and various inflammatory and metabolic parameters in a Japanese population. J Atheroscler Thromb. 2009;16(2):77-82.

23. Guo X, Ko JK, Mei QB, Cho CH. Aggravating effect of cigarette smoke exposure on experimental colitis is associated with leukotriene $\mathrm{B}(4)$ and reactive oxygen metabolites. Digestion. 2001;63(3):180-187.

24. Teramoto T, Sasaki J, Ueshima H, et al. Japan Atherosclerosis Society (JAS) Committee for Epidemiology and Clinical Management of Atherosclerosis. Diagnostic criteria for dyslipidemia. Executive summary of Japan Atherosclerosis Society (JAS) guideline for diagnosis and prevention of atherosclerotic cardiovascular diseases for Japanese. J Atheroscler Thromb. 2007;14(4):155-158.

25. Ogihara T, Kikuchi K, Matsuoka H, et al. Japanese Society of Hypertension Committee. The Japanese Society of Hypertension guidelines for the management of hypertension (JSH 2009). Hypertens Res. 2009;32(1):3-107.

26. Sugiura T, Kondo T, Kureishi-Bando Y, et al. Nifedipine improves endothelial function: role of endothelial progenitor cells. Hypertension. 2008;52(3):491-498.

27. Hill JM, Zalos G, Halcox JP, et al. Circulating endothelial progenitor cells, vascular function, and cardiovascular risk. $N$ Engl J Med. 2003;348(7):593-600.

28. Heiss C, Keymel S, Niesler U, Ziemann J, Kelm M, Kalka C. Impaired progenitor cell activity in age-related endothelial dysfunction. $J \mathrm{Am}$ Coll Cardiol. 2005;45(9):1441-1448.

29. Brinkley TE, Nicklas BJ, Kanaya AM, et al. Plasma oxidized low-density lipoprotein levels and arterial stiffness in older adults: the health, aging, and body composition study. Hypertension. 2009;53(5):846-852.

30. Tanaga $\mathrm{K}$, Bujo $\mathrm{H}$, Inoue $\mathrm{M}$, et al. Increased circulating malondialdehyde-modified LDL levels in patients with coronary artery diseases and their association with peak sizes of LDL particles. Arterioscler Thromb Vasc Biol. 2002;22(4):662-666.

31. Marchesi E, Martignoni A, Salvini M, et al. Carotid intima-media thickening and in vivo LDL oxidation in patients with essential hypertension. J Hum Hypertens. 1996;10(9):577-582.

32. Miyazaki T, Shimada K, Sato O, et al. Circulating malondialdehydemodified LDL and atherogenic lipoprotein profiles measured by nuclear magnetic resonance spectroscopy in patients with coronary artery disease. Atherosclerosis. 2005;179(1):139-145.

33. Doust JA, Pietrzak E, Dobson A, Glasziou P. How well does B-type natriuretic peptide predict death and cardiac events in patients with heart failure: Systematic review. BMJ. 2005;330(7492):625-634.

34. Takase H, Toriyama T, Sugiura T, et al. Brain natriuretic peptide detects cardiac abnormalities in mass screening. Eur J Clin Invest. 2007;37(4):25-62.

\section{Dovepress}

metabolic disorders, particularly diabetes. This journal is indexed on PubMed Central and MedLine. The manuscript management system is completely online and includes a very quick and fair peer-review system, which is all easy to use. Visit http://www.dovepress.com/ testimonials.php to read real quotes from published authors. 